\title{
A rare variant of the TYK2 gene is confirmed to be associated with multiple sclerosis
}

\author{
Inger-Lise Mero ${ }^{1,2}$, Åslaug R Lorentzen ${ }^{2,3}$, Maria Ban ${ }^{4}$, Cathrine Smestad ${ }^{1}$, Elisabeth G Celius ${ }^{1}$, \\ Jan H Aarseth ${ }^{5}$, Kjell-Morten Myhr ${ }^{5}$, Jenny Link ${ }^{6}$, Jan Hillert ${ }^{6}$, Tomas Olsson ${ }^{7}$, Ingrid Kockum ${ }^{7}$, \\ Thomas Masterman $^{6}$, Annette Bang Oturai ${ }^{8}$, Helle Bach Søndergaard ${ }^{8}$, Finn Sellebjerg ${ }^{8}$, Janna Saarela ${ }^{9}$, \\ Anu Kemppinen ${ }^{9}$, Irina Elovaara ${ }^{10}$, Anne Spurkland ${ }^{11}$, Frank Dudbridge ${ }^{12}$, Benedicte A Lie ${ }^{2}$ \\ and Hanne F Harbo ${ }^{1,3}$
}

\begin{abstract}
A rare functional variant within the TYK2 gene (rs34536443) has been reported as protective in multiple sclerosis (MS) in recent studies. However, because of the low frequency of the minor allele (minor allele frequency=0.04), genome-wide significant association has been hard to establish. We genotyped 5429 Nordic MS cases and 6167 healthy controls for this TYK2 non-synonymous single-nucleotide polymorphism (ns-SNP), and combined the Nordic genotype data with raw genotypes from previous studies. The combined Nordic analysis showed significant association with MS $\left(P=5 \times 10^{-4}\right.$, odds ratio (OR) 0.78 ), and by mega-analysis of 10642 MS patients, 10620 controls and $2110 \mathrm{MS}$ trios, the association at genome-wide significance level $\left(P=5.08 \times 10^{-9}\right.$, OR 0.77$)$ was shown.
\end{abstract}

European Journal of Human Genetics (2010) 18, 502-504; doi:10.1038/ejhg.2009.195; published online 4 November 2009

Keywords: TYK2; MS; ns-SNP; autoimmune disease

\section{INTRODUCTION}

Multiple sclerosis (MS) is a chronic inflammatory disorder of the central nervous system of possible autoimmune etiology, and its association with the HLA complex has been known for many years. ${ }^{1}$ As for most immune-mediated diseases, MS has a proposed complex genetic etiology, but the search for non-HLA susceptibility genes has proven difficult until the introduction of genome-wide association studies (GWASs). ${ }^{2,3}$ Since 2007, four GWASs in MS have identified several common susceptibility variants outside the HLA complex, ${ }^{4-7}$ many of which are shared by other autoimmune diseases. However, low-frequency alleles, set to a minor allele frequency (MAF) below 0.05 in this study, are seldom included in such studies because of the difficulties in detecting moderate genetic effects at this frequency level. ${ }^{8}$

A rare variant of the TYK2 gene ( $\mathrm{rs} 34536443, \mathrm{MAF}=0.04$ ), located on chromosome 19p13, was first identified as a possible susceptibility factor in the combined analysis of autoimmune inflammatory thyroiditis, ankylosing spondylitis and MS in an association scan of 14500 non-synonymous single-nucleotide polymorphisms (ns-SNPs) carried out by the Wellcome Trust Case Control Consortium (WTCCC) and the Australo-Anglo-American Spondylitis Consortium (TASC) in 2007..$^{5}$ The associated variant encodes a proline (major allele) to alanine substitution in exon 21 of the TYK2 gene. The TYK2 association in MS was recently substantiated in a replication analysis by Ban $e t$ a $l^{9}$ in a sample set of 4234 MS patients, 2983 controls and 2053 trio families from Australia, Belgium, Norway, United Kingdom and the United States of America $\left(P=2.7 \times 10^{-6}\right)$. However, because of the low frequency of the minor allele, conclusive association with MS at genome-wide significance level $\left(P \leqslant 5 \times 10^{-7}\right)$ was not established.

\section{MATERIALS AND METHODS}

In this Nordic collaboration, samples from 5429 MS cases and 6167 healthy controls were collected in Denmark, Finland, Norway and Sweden (sample distribution shown in Table 1). Patients were diagnosed with MS according to Poser and/or McDonald criteria. ${ }^{10,11}$ The Norwegian samples did not overlap with the samples included in the replication study by Ban et al. ${ }^{9}$ Informed consent from all participants and local ethical approvals were obtained. The samples were genotyped for the TYK2 rs34536443 SNP, performed using TaqMan chemistry on an ABI7900 HT real-time PCR system (Applied Biosystems, Foster city, CA, USA) or on a Sequenom iPLEX platform (Sequenom Inc., San Diego, CA, USA).

The statistical analyses of the Nordic genotype data were carried out using PLINK v1.05 (http://pngu.mgh.harvard.edu/purcell/plink/). ${ }^{12}$ The homogeneity of the odds ratios (OR) from the four Nordic populations was tested using the Breslow-Day test, and a combined analysis was performed using the Cochran-Mantel-Haenszel (CMH) test. The Nordic genotype data were then

${ }^{1}$ Department of Neurology, Oslo University Hospital, Ullevål, Oslo, Norway; ${ }^{2}$ Institute of Immunology, Oslo University Hospital, Rikshospitalet, Oslo, Norway; ${ }^{3}$ Department of Neurology, Oslo University Hospital, Faculty Division Ullevål, University of Oslo, Oslo, Norway; ${ }^{4}$ Department of Clinical Neuroscience, University of Cambridge, Addenbrooke's Hospital, Cambridge, UK; ${ }^{5}$ Department of Neurology, Haukeland University Hospital, The Norwegian Multiple Sclerosis Registry and Biobank, Bergen, Norway; ${ }^{6}$ Department of Clinical Neuroscience, Center for Molecular Medicine, The Multiple Sclerosis Research Group, Karolinska Institutet, Stockholm, Sweden; ${ }^{7}$ Neuroimmunology Unit, Department of Clinical Neuroscience and Center for Molecular Medicine, Karolinska Institutet at Karolinska University Hospital, Solna, Sweden; ${ }^{8}$ The Danish Multiple Sclerosis Research Center, Department of Neurology, Copenhagen University Hospital, Copenhagen, Denmark; 9 Institute for Molecular Medicine Finland FIMM, University of Helsinki, Helsinki, Finland; ${ }^{10}$ Neuroimmunology Unit, Medical School, University of Tampere and Department of Neurology, Tampere University Hospital, Finland; ${ }^{11}$ Institute of Basal Medical Science, University of Oslo, Oslo, Norway; ${ }^{12 M R C ~ B i o s t a t i s t i c s ~ U n i t, ~ C a m b r i d g e, ~ U K ~}$

Correspondence: Dr I-L Mero, Department of Neurology, Oslo University Hospital, Ullevål, N-0407 Oslo, Norway. Tel: +47 23073813; Fax: +47 23073510;

E-mail: ilmero@rr-research.no

Received 1 July 2009; revised 17 September 2009; accepted 22 September 2009; published online 4 November 2009 
Table 1 Association testing of the TYK 2 gene SNP rs34536443

\begin{tabular}{|c|c|c|c|c|c|c|c|}
\hline Population & Cases (n) & Controls (n) & & MAF cases & MAF controls & OR $(95 \% \mathrm{Cl})$ & P-value \\
\hline Danish & 1112 & 1147 & & 0.036 & 0.042 & $0.85(0.63-1.16)$ & 0.30 \\
\hline Finnish & 810 & 1079 & & 0.021 & 0.031 & $0.67(0.44-1.02)$ & 0.06 \\
\hline Norwegian I & 1396 & 2064 & & 0.042 & 0.051 & $0.82(0.65-1.03)$ & 0.08 \\
\hline Swedish & 2111 & 1877 & & 0.032 & 0.042 & $0.76(0.60-0.96)$ & 0.02 \\
\hline Combined analysis & Cases (n) & Controls (n) & Trios & & & OR $(95 \% \mathrm{Cl})$ & P-value \\
\hline Nordic combined ${ }^{a}$ & 5429 & 6167 & & & & $0.78(0.68-0.90)$ & 0.00054 \\
\hline Mega-analysis ${ }^{\mathrm{b}}$ & 10642 & 10620 & 2110 & & & $0.77(0.70-0.84)$ & 5.083E-09 \\
\hline
\end{tabular}

Abbreviations: $\mathrm{Cl}$, confidence interval; MAF, minor allele frequency; OR, odds ratio.

aNordic combined analysis includes data from the Danish, Finnish, Norwegian I and Swedish sample sets, genotyped in this study.

${ }^{\mathrm{b}} \mathrm{A}$ mega-analysis of data from the Nordic analysis combined with the data from the United Kingdom, Belgian, Norwegian II, Australian and the United States sample sets, as published by Ban et al.

combined with the data from the replication analysis performed by Ban $e t a l^{9}$ and the original WTCCC/TASC study. ${ }^{5}$ This mega-analysis, comprising 10642 MS patients, 10620 controls and 2110 MS trios, was performed using likelihood ratio chi-square testing with nationality set as a discrete confounder in Unphased v3.1.3 statistical software (http://www.mrc-bsu.cam.ac.uk/personal/ frank/software/unphased/). ${ }^{13}$

\section{RESULTS}

In all Nordic populations, the frequency of the minor allele $\mathrm{C}$ of the ns-SNP analyzed was lower in cases compared with controls (Table 1). Genotyping success rate was above $97 \%$, and there was no significant deviation from the Hardy-Weinberg equilibrium $(P \geqslant 0.05)$. The Breslow-Day test excluded significant heterogeneity within the Nordic populations $(P=0.8)$, allowing for a combined Nordic analysis $(n=5429 \mathrm{MS}$ cases and 6167 healthy controls) that revealed significant association $\left(P=5 \times 10^{-4}\right.$, OR $0.78,95 \%$ CI $\left.0.68-0.90\right)$. In the analysis of the Nordic data combined with the raw genotype data from the replication analysis by Ban et al ${ }^{9}$ and the original WTCCC/TASC study $(n=10642 \mathrm{MS}$ patients, 10620 controls and $2110 \mathrm{MS}$ trios $),{ }^{5}$ the Breslow-Day test yielded a $P$-value of 0.05 ; thus, the $\mathrm{CMH}$ test was replaced by a likelihood ratio test, accounting for population heterogeneity. In this mega-analysis, association with the TYK2 SNP reached a convincing level of genome-wide significance $\left(P=5.08 \times 10^{-9}\right.$, OR $0.77,95 \%$ CI $0.70-0.84$ ).

\section{DISCUSSION}

GWASs are powerful tools to detect common genetic susceptibility variants in complex diseases. However, low-frequency susceptibility alleles (MAF <0.05), which exert a modest effect, are harder to establish as large sample sets are required to show association at genome-wide significance level $\left(P \leqslant 5 \times 10^{-7}\right)$. Even if we saw the same trend in all Nordic populations toward a protective effect of the minor allele of the analyzed SNP, analysis of more than 5000 patients and 6000 controls was not sufficient to reach genome-wide significant association. More than 10000 cases and 10000 controls are estimated to be required to have $80 \%$ power to detect associations of similar frequencies and effects with genome wide-significance (Quanto power calculator; http://hydra.usc.edu/gxe/). Indeed, this was achieved by increasing our sample size to $10642 \mathrm{MS}$ patients, 10620 controls and 2110 MS trios, whereby association reached $P=5.08 \times 10^{-9}$.

Low-frequency SNPs, as analyzed in this study, have often not been selected for genome-wide screens, in which the focus traditionally has been on common variants. More than 1500 published genetic susceptibility variants in complex traits are now listed in the
Office of Publication Genomics: A catalogue of published GWASs (www.genome.gov, accessed 23 June 2009), but low-frequency risk alleles make up less than $3 \%$ of the total associations reported. However, the WTCCC ns-SNP study had a different approach, that is, to scan only markers that cause alteration in the encoded protein sequence. $^{5}$ This also implies that the associated TYK2 ns-SNP rs34536443 might have a direct functional significance. TYK2 is a proximal tyrosine kinase in the STAT signaling pathway that is important for signaling by type I interferons and induction of Th1 cell differentiation upon antigen stimulation of dendritic cells. ${ }^{14}$ The rs34536443 alters the amino acid sequence at position 1104 within the kinase domain of the TYK2 protein, and the protective minor allele, encoding alanine, is predicted to give a less efficient variant of TYK2. ${ }^{9}$ Interestingly, another functional SNP in TYK2 was recently confirmed to confer susceptibility to systemic lupus erythematosus (SLE). ${ }^{15}$ Comparing the MS- and SLE-associated TYK2 SNPs, that is, rs34536443 with rs2304256 in Haploview v.4.1 (using the CEU individuals from the HapMap project phase 1, 2 and 3 (http://www. hapmap.org/ and http://www.ncbi.nlm.nih.gov/)), there was no evidence of linkage disequilibrium (LD) between these SNPs $\left(r^{2}=0.044\right) .{ }^{16}$ Further analysis is required to see whether these SNPs are associated with both MS and SLE; however, should this be the case, the low LD suggests that these functional variants within the TYK2 gene confer independent effects or point toward a third common causal variant.

In conclusion, we confirm a protective effect of a rare variant in the TYK2 gene in MS. The study illustrates the requirement of large sample sizes for confirmation of low-frequency susceptibility alleles, and to our knowledge, this is the first low-frequency allele shown to be associated with MS at a genome-wide significance level. Although GWASs are establishing associations with more common variants, many of the rare variants are yet to be detected. The next generation of sequencing methods will hopefully facilitate the revelation of these SNPs.

\section{CONFLICT OF INTEREST}

The authors declare no conflict of interest.

\section{ACKNOWLEDGEMENTS}

We thank MS patients and healthy controls for their participation in the study. We gratefully acknowledge the contribution made by the various members of the International Multiple Sclerosis Genetics Consortium (IMSGC) (https:// www.imsgc.org), in particular, Dr Stephen Sawcer, as well as the Wellcome Trust Case Control Consortium (WTCCC), who kindly supplied their data 
for inclusion in this analysis. The Oslo MS genetics group is supported by grants from The Research Council of Norway; Scientific Advisory Council Ullevål, Oslo University Hospital; South-Eastern Norway Regional Health Authority, Norwegian Foundation for Health and Rehabilitation and Odd Fellow MS society. Contributors to the collection of samples and clinical data in the Norwegian MS Registry and Biobank are acknowledged for their contributions. The Norwegian MS Registry and Biobank is supported by The Research Council of Norway, Haukeland University Hospital and Western Norway Regional Health Authority. The Institute of Medical Genetics, Ulleval, Oslo University Hospital, and the Norwegian Bone Marrow Donor Registry, Rikshospitalet, Oslo University Hospital are acknowledged for providing Norwegian controls. The Finnish MS group is supported by Neuropromise EU grant LSHM-CT-2005-018637, The Center of Excellence for Disease Genetics of the Academy of Finland, the Sigrid Juselius Foundation, Helsinki University Central Hospital Research Foundation, and the Helsinki Biomedical Graduate School (AK). The MGEN consortium neurologists Dr Keijo Koivisto, Dr Tuula Pirttilä, Dr Mauri Reunanen, and Dr Pentti Tienari are acknowledged for the Finnish MS patient samples and the clinical data. The Danish Multiple Sclerosis Society is acknowledged for financial support of the Danish MS research. The Swedish group was funded by the Bibbi and Nils Jensen Foundation, Montel Williams foundation and the Söderbergs foundation, the Swedish Research Council, Karolinska Institutet, and the 6th Framework Program of the European Union, NeuroproMiSe (LSHM-CT-2005-018637).

1 Jersild C, Svejgaard A, Fog T: HL-A antigens and multiple sclerosis. Lancet 1972; 1: $1240-1241$.

2 Oksenberg JR, Barcellos LF: Multiple sclerosis genetics: leaving no stone unturned. Genes Immun 2005; 6: 375-387.
3 Sawcer S: The complex genetics of multiple sclerosis: pitfalls and prospects. Brain 2008; 131: 3118-3131.

4 International Multiple Sclerosis Genetics Consortium (IMSGC): Risk alleles for multiple sclerosis identified by a genomewide study. N Engl J Med 2007; 357: 851-862.

5 Wellcome Trust Case Control Consortium and The Australo-Anglo-American Spondylitis Consortium: Association scan of 14500 nonsynonymous SNPs in four diseases identifies autoimmunity variants. Nat Genet 2007; 39: 1329-1337.

6 Baranzini SE, Wang J, Gibson RA et al: Genome-wide association analysis of susceptibility and clinical phenotype in multiple sclerosis. Hum Mol Genet 2009; 18: 767-778.

7 The Australia and New Zealand Multiple Sclerosis Genetics Consortium (ANZgene): Genome-wide association study identifies new multiple sclerosis susceptibility loci on chromosomes 12 and 20. Nat Genet 2009; 41: 824-828.

8 Wang WY, Barratt BJ, Clayton DG. et al: Genome-wide association studies: theoretical and practical concerns. Nat Rev Genet 2005; 6: 109-118.

9 Ban M, Goris A, Lorentzen AR et al: Replication analysis identifies TYK2 as a multiple sclerosis susceptibility factor. Eur J Hum Genet 2009; 17: 1309-1313.

10 McDonald WI, Compston A, Edan G et al: Recommended diagnostic criteria for multiple sclerosis: guidelines from the International Panel on the diagnosis of multiple sclerosis. Ann Neurol 2001; 50: 121-127.

11 Poser CM, Paty DW, Scheinberg L et al: New diagnostic criteria for multiple sclerosis: guidelines for research protocols. Ann Neurol 1983; 13: 227-231.

12 Purcell S, Neale B, Todd-Brown K et al: PLINK: a tool set for whole-genome association and population-based linkage analyses. Am J Hum Genet 2007; 81: 559-575.

13 Dudbridge F: Likelihood-based association analysis for nuclear families and unrelated subjects with missing genotype data. Hum Hered 2008; 66: 87-98.

14 Tokumasa N, Suto A, Kagami S et al: Expression of Tyk2 in dendritic cells is required for IL-12, IL-23, and IFN-gamma production and the induction of Th1 cell differentiation. Blood 2007; 110: 553-560.

15 Suarez-Gestal M, Calaza M, Endreffy E et al: Replication of recently identified systemic lupus erythematosus genetic associations: a case control study. Arthritis Res Ther 2009; 11: R69.

16 Barrett JC, Fry B, Maller J et al: Haploview: analysis and visualization of LD and haplotype maps. Bioinformatics 2005; 21: 263-265. 